Journal for ImmunoTherapy of Cancer

\title{
Initiation of immunotherapy with activated natural killer cells and anti- GD2 antibody dinutuximab prior to resection of primary neuroblastoma prolongs survival in mice
}

\author{
Michael John Zobel (D) , ${ }^{1}$ Abigail K Zamora, ${ }^{1}$ Hong-wei Wu, ${ }^{2}$ Jianping Sun, ${ }^{2}$ \\ Danny Lascano, ${ }^{1}$ Jemily Malvar, ${ }^{2}$ Larry Wang, ${ }^{3}$ Michael A Sheard (D) , \\ Robert C Seeger, ${ }^{2}$ Eugene S Kim (D) ${ }^{1,4}$
}

To cite: Zobel MJ, Zamora AK, Wu $\mathrm{H}$, et al. Initiation of immunotherapy with activated natural killer cells and antiGD2 antibody dinutuximab prior to resection of primary neuroblastoma prolongs survival in mice. Journal for ImmunoTherapy of Cancer 2020;8:e001560. doi:10.1136/ jitc-2020-001560

Accepted 24 November 2020

Check for updates

(C) Author(s) (or their employer(s)) 2020. Re-use permitted under CC BY-NC. No commercial re-use. See rights and permissions. Published by BMJ.

For numbered affiliations see end of article.

\section{Correspondence to}

Dr Eugene S Kim, Division of Pediatric Surgery, Children's Hospital Los Angeles, Los Angeles, CA, USA;

eugeneskim@chla.usc.edu

\section{ABSTRACT}

Background Immunotherapy with anti-disialoganglioside dinutuximab has improved survival for children with high-risk neuroblastoma (NB) when given after induction chemotherapy and surgery. However, disease recurrence and resistance persist. Dinutuximab efficacy has not been evaluated when initiated before primary tumor removal. Using a surgical mouse model of human NB, we examined if initiating dinutuximab plus ex vivo-activated natural killer (aNK) cells before resection of the primary tumor improves survival.

Methods In vitro, human NB cells (SMS-KCNR-Fluc, CHLA-255-Fluc) were treated with dinutuximab and/or aNK cells and cytotoxicity was measured. In vivo, NB cells (SMS-KCNR-Fluc, CHLA-255-Fluc, or COG-N-415x PDX) were injected into the kidney of NOD-scid gamma mice. Mice received eight intravenous infusions of aNK cells plus dinutuximab beginning either 12 days before or 2 days after resection of primary tumors. Tumors in control mice were treated by resection alone or with immunotherapy alone. Disease was quantified by bioluminescent imaging and survival was monitored. aNK cell infiltration into primary tumors was quantified by flow cytometry and immunohistochemistry at varying timepoints.

Results In vitro, aNK cells and dinutuximab were more cytotoxic than either treatment alone. In vivo, treatment with aNK cells plus dinutuximab prior to resection of the primary tumor was most effective in limiting metastatic disease and prolonging survival. aNK cell infiltration into xenograft tumors was observed after 1 day and peaked at 5 days following injection.

Conclusion Dinutuximab plus aNK cell immunotherapy initiated before resection of primary tumors decreases disease burden and prolongs survival in an experimental mouse model of NB. These findings support the clinical investigation of this treatment strategy during induction therapy in patients with high-risk NB.

\section{INTRODUCTION}

Approximately $80 \%$ of children with highrisk neuroblastoma (NB) achieve remission after induction chemotherapy, surgical resection of the primary tumor, local irradiation, myeloablative chemotherapy with autologous hematopoietic stem cell transplantation, and immunotherapy with antidisialoganglioside (GD2) dinutuximab, granulocyte-macrophage colony-stimulating factor (GM-CSF), and interleukin-2 (IL). However, $55 \%$ of patients will subsequently succumb from progression of metastatic disease. Post-consolidation immunotherapy with dinutuximab has been shown to increase 2-year event-free and overall survival and is now the standard treatment for children with high-risk disease. ${ }^{12}$ In our previous work, we showed that the combination of adoptively transferred ex vivo-activated natural killer (aNK) cells and dinutuximab improves survival and reduces metastatic burden in a NB mouse model of minimal residual disease (MRD) compared with either treatment alone. ${ }^{34}$ This has provided preclinical support for multiple phase I clinical trials exploring the benefit of treating patients with refractory or relapsed NB with aNK cells and anti-GD2 antibody (NCT02573896, NCT01576692, NCT00877110, NCT02650648).

The efficacy of initiating immunotherapy with dinutuximab plus adoptively transferred aNK cells prior to surgical resection of primary NB tumors has not been addressed in the literature. We developed a model of neuroblastoma in immunodeficient NODscid gamma (NSG) mice in which human NB cells are injected beneath the capsule of the left kidney. Previously, we demonstrated that NSG mice undergoing gross total resection of tumors en bloc with the entire kidney 7 days after tumor cell injection reliably achieved 
MRD and consistently developed disease in the liver and bone marrow, both clinically relevant sites of metastasis. In the current study, we delayed resection of the xenografted primary tumor until 19 days after tumor cell injection to test the hypothesis that initiating immunotherapy with dinutuximab and adoptively transferred aNK cells before surgical resection would enhance reduction of disease burden and improve survival.

\section{METHODS}

\section{NB cell lines and patient-derived xenograft}

Human NB cell lines SMS-KCNR and CHLA-255, and patient-derived xenograft (PDX) COG-N-415x cells, were derived from patients with progressive disease. CHLA-255 and SMS-KCNR cells were obtained from the Children's Oncology Group (COG) Cell Culture and Xenograft Repository (www.COGcell.org). The firefly luciferase (Fluc) gene was transduced into SMS-KCNR cells and CHLA-255 cells using a lentivirus vector, as previously described. ${ }^{35}$ SMS-KCNR-Fluc was maintained in RPMI1640, and CHLA-255-Fluc was maintained in Iscove's modified Dulbecco's medium, both with $10 \%$ fetal bovine serum. PDX was cryopreserved at low passage (less than 5) and expanded in vivo by subcutaneous injection into NSG mice. SMS-KCNR cells have genomic amplification of MYCN, while CHLA-255 cells overexpress c-Myc protein and lack MYCN amplification. COG-N-415x cells have amplification of MYCN and mutation of ALK (F1174L; provided by Dr Patrick Reynolds, www.COGcell.org). All three express a high level of GD2 on the tumor cell surface $^{6}$. All cell lines were screened routinely for $M y c o-$ plasma, and donor cell identity was authenticated by short tandem repeat multiplex assay using the AmpFLSTR Identifiler PCR Amplification Kit (Applied Biosystems; catalog no. 4322288).

NK cell activation and propagation aNK cells from peripheral blood mononuclear cells of healthy human donors were propagated and activated ex vivo using irradiated K562-mbIL21 stimulatory cells and IL-2 (Peprotech, Rocky Hill, New Jersey, USA), as previously described. ${ }^{378}$ EasySep magnet-activated cell sorting was used to positively select T cells on day 7 of cultures, enabling the enrichment of NK cells (StemCell Technologies, Vancouver, California, USA). aNK cells, which were greater than $99 \%$ CD56 and CD16 positive by flow cytometry after 21 days of ex vivo propagation, were aliquoted and viably frozen in cryoprotective medium (Lonza Bioscience, Morrisville, North Carolina, USA).

\section{Cytotoxicity assay}

Cryopreserved K562-mbIL21-expanded aNK cells were cultured in 10\% RPMI-1640 media with IL-2 $(10 \mathrm{ng} / \mathrm{mL})$ for 48 hours. $1.5 \times 10^{4} \mathrm{NB}$ cells (SMS-KCNR-Fluc, CHLA255-Fluc) were seeded per well in a 96-well plate. NB cells were either untreated or treated with dinutuximab alone, aNK cells alone, or aNK cells plus dinutuximab. Dinutuximab was added at a concentration of $100 \mathrm{ng} / \mathrm{mL}$.
$1.5 \times 10^{4}$ aNK cells were added at an effector:target $(\mathrm{E}: \mathrm{T})$ ratio of 1:1. IL-2 was added to wells containing aNK cells to create a final concentration of $5 \mathrm{ng} / \mathrm{mL}$. The plate was incubated at $37^{\circ} \mathrm{C}$ in $5 \% \mathrm{CO}_{2}$ for 6 hours, and survival of NB cells was assessed by luminescence after addition of $50 \mathrm{ng}$ of luciferin (Promega, Madison, Wisconsin, USA). Cell survival for all groups was normalized to untreated cell survival.

\section{Tumor cell injection, tumor resection, and treatment of NSG mice}

Eight-week to 12-week-old NSG mice, which lack NK, B, and $\mathrm{T}$ cells, were used for all in vivo experiments. One day prior to tumor cell injection, mice were given $250 \mathrm{cGy}$ of whole body irradiation. NB cell viability was confirmed using trypan blue staining prior to injection. $1 \times 10^{6} \mathrm{NB}$ cells (SMS-KCNR-Fluc, CHLA-255-Fluc, or COG-N$415 \mathrm{x}$ ) suspended in $0.1 \mathrm{~mL}$ of phosphate buffered saline (PBS) (Omega Scientific, PB-24) were surgically injected beneath the renal capsule of left kidneys of anesthetized mice, as per our previously described model. ${ }^{49}$ In total, 125 mice were injected for this experiment: 58 with SMSKCNR-Fluc cells, 32 with CHLA-255-Fluc cells, 35 with COG-N-415x cells. Groups of mice were age and sex matched. Mice injected with luciferase-expressing cell lines were assessed by bioluminescent imaging using a Xenogen IVIS 100 instrument (IVIS Lumina XR System, Caliper Life Sciences) on day 7 after NB cell injection, to allow equal stratification of mice based on starting tumor signal.

Treatments with dinutuximab $(15 \mu \mathrm{g} /$ mouse $)$ and aNK cells $\left(1 \times 10^{7} /\right.$ mouse, immediately after thawing), plus recombinant human IL-2 $(2 \mu \mathrm{g} /$ mouse; Peprotech; catalog 200-02) and IL-15 (5 $\mathrm{g} /$ mouse; generously provided by the NCI BRB Preclinical Repository) were infused intravenously via tail vein injection twice weekly for eight treatments per our previously published protocol (figure 1). ${ }^{3}$ As an activator of NK cells, IL-15 was included in this experiment to maintain consistency with the treatment protocols from our previously published preclinical experiments and for the purposes of direct comparison. ${ }^{4-6}$

Mice receiving immunotherapy before surgery began treatment on day 7 after tumor cell injection (12 days before tumor resection), while mice receiving immunotherapy only after surgery began treatment on day 21 (2 days after tumor resection). Control groups included mice that underwent resection without immunotherapy and mice that received immunotherapy alone beginning on either day 7 or day 21 without resection. Surgical resection of primary tumor was performed on day 19 under general anesthesia. Left kidneys were exposed via the same incision and the renal hilum was ligated; the xenografted tumor and left kidney were resected en bloc, followed by closure of the muscle and skin layers, as per the first operation. ${ }^{49}$ Mice with luciferase-expressing cell lines underwent weekly bioluminescent imaging to measure disease burden. As per the data from our previously described 


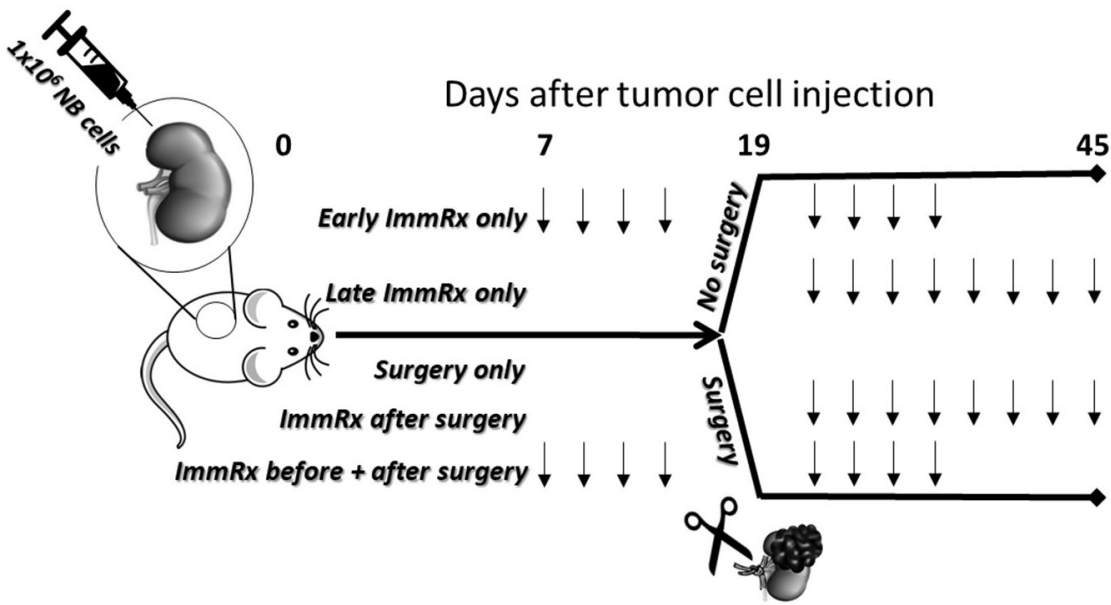

Figure 1 Schematic diagram of the in vivo experimental protocol. $1 \times 10^{6}$ human neuroblastoma (NB) cells were injected beneath the left renal capsule of NOD-scid gamma mice on day 0 . Mice receiving immunotherapy received dinutuximab (15 $\mu$ g)

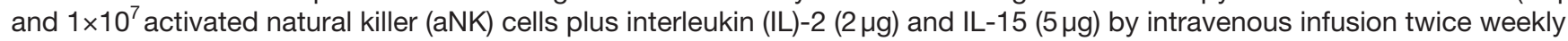
for 4 weeks. Each arrow indicates one treatment. 'Surgery' indicates resection of the primary tumor which, when performed, occurred on day 19. Mice in the 'surgery only' group received no immunotherapy and only underwent resection. ImmRx, immunotherapy.

model, tumor recurrence following resection occurs at the primary tumor site, as well as within the liver and bone marrow. ${ }^{9}$ Total flux (photons/second) from each mouse was quantified using Living Image software (Living Image V.4.3.1, Caliper Life Sciences) and compared with pretreatment bioluminescence. Mice were also monitored for survival and were euthanized when IACUC criteria for euthanasia were met, which included lethargy, poor grooming, weight loss, and/or hind-limb paralysis.

\section{In vivo infiltration of xenograft tumors by NK cells}

Human NB xenograft tumors were established in NSG mice as described above. Tumor-bearing mice were treated once via tail vein injection with $1 \times 10^{7}$ aNK cells (immediately after thawing) plus dinutuximab and human recombinant IL-2 and IL-15, with the same dosages as described above, on day 19, 24, or 28 after NB cell injection, representing 10 days, 5 days, or 1 day prior to tumor resection. Control mice were not treated with aNK cells. Tumors were resected on day 29 after NB cell injection, were dissociated into single-cell suspensions using the MACS Tumor Dissociation Kit and a gentleMACS Tissue Dissociator (Miltenyi Biotec), and were analyzed by flow cytometry.

After washing twice with fluorescence-activated cell sorting (FACS) buffer (PBS+0.5\% PharmingenStain Buffer $+1 \mathrm{mM}$ EDTA), cells were treated with Human TruStain FcX, an Fc receptor blocker (Biolegend, $\# 422302$ ), for $7 \mathrm{~min}$ at room temperature to minimize non-specific antibody binding. Cells then were stained for 1 hour in the dark at $4^{\circ} \mathrm{C}$ for cell surface markers: GD2, an NB cell marker, and human CD45, a pan-leukocyte marker. Anti-GD2 antibody was conjugated to APC (Clone 14G2a, Biolegend, \#357306) and anti-human CD45 antibody to AlexaFluor 488 (Clone 2D1, Biolegend, \#368536). Isotype controls were used for each fluorochrome: (1)
APC (Mouse IgG1, BD Pharmingen, \#340754) and (2) AlexaFluor 488 (Mouse IgG1, BioLegend, \#400129). After a final wash with FACS buffer, samples were resuspended and stained with DAPI (4',6-diamidino-2-phenylindole) nucleic acid viability dye to facilitate dead cell exclusion (Sigma-Aldrich, \#D9542). Cell suspensions were passed through a 35-micron nylon strainer prior to analysis by flow cytometry using a BD LSRII (BD Biosciences, San Jose, California, USA) and BD FACSDiva Software (V.6.1.3, BD Biosciences, San Jose, California, USA).

Infiltration of xenografted tumors by aNK cells was confirmed by immunohistochemical staining of tumors resected from mice 29 days after injection of $1 \times 10^{6} \mathrm{NB}$ cells that were preserved in formalin at $4^{\circ} \mathrm{C}$. Tumor tissue was cut into $5 \mu \mathrm{m}$ sections and stained with mouse anti-human CD16 monoclonal antibody (Bio-Rad, \#MCA2537T) by the Department of Pathology at Children's Hospital Los Angeles (Los Angeles, California, USA). Sections were evaluated for the presence of aNK cells with the tumors by Dr LW, attending pathologist, who specializes in NB.

\section{Statistical analyses}

Data from treatment groups in both the in vitro cytotoxicity assay and the NK infiltration study were compared using Student's t-tests. In vivo weekly bioluminescent flux measurements were adjusted to baseline observations and the area under the curve (AUC) was calculated. The AUC was $\log _{10}$ transformed to normalize the data, and linear regression was used to assess the treatment effects on metastatic burden $\left(\log _{\mathrm{AUC}}\right)$. Survival time was defined as the number of days from the day of NB cell injection to the day each mouse was either found dead or met IACUC criteria for euthanasia. Differences in survival between groups were analyzed by linear regression. Analyses were performed using Statistical Package for the Social Sciences V.17.0 (SPSS) and Stata V.11 (StataCorp LP). 
A
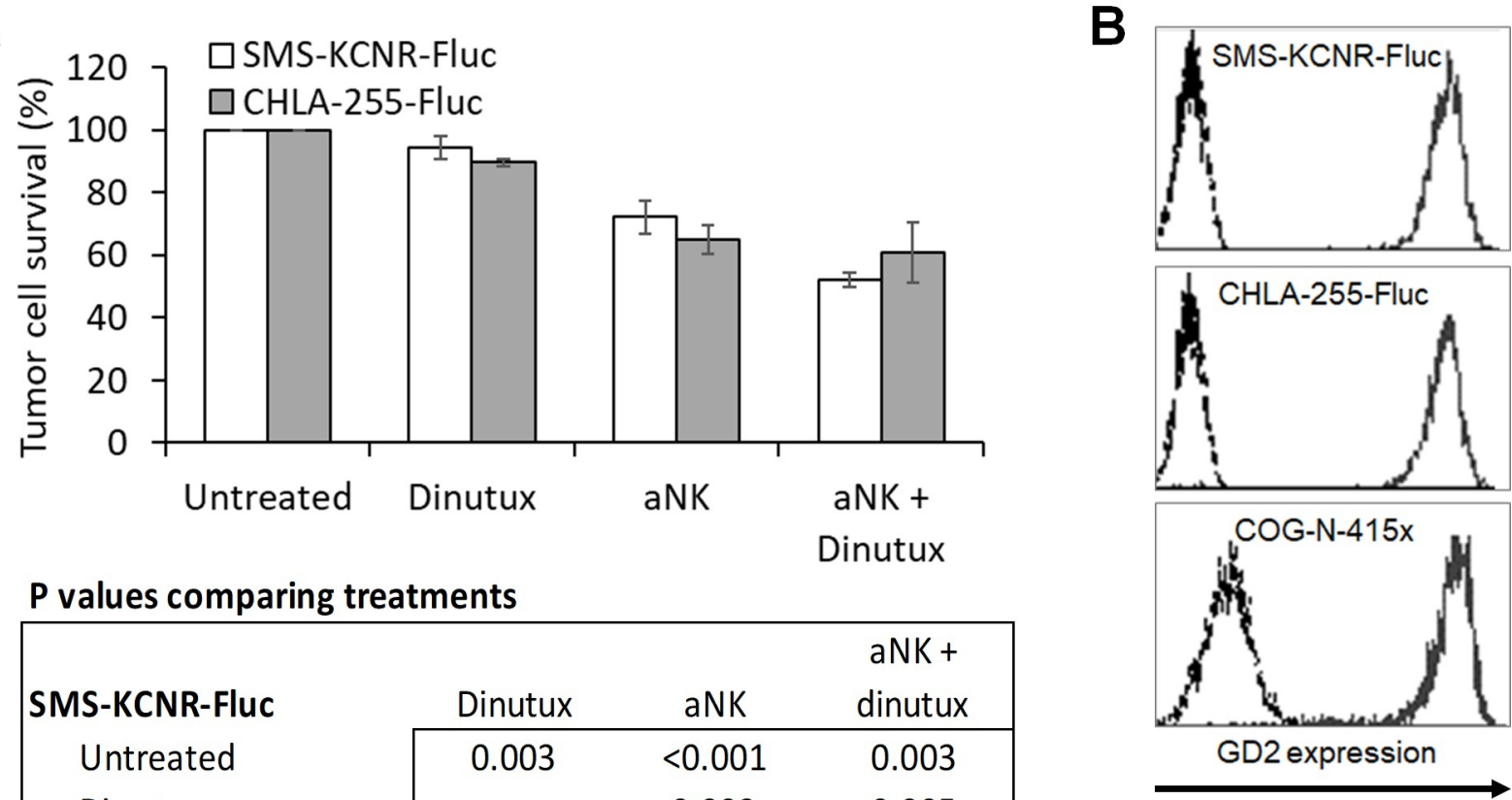

P values comparing treatments

\begin{tabular}{|lccc|}
\hline \multicolumn{1}{l}{ SMS-KCNR-Fluc } & \multicolumn{3}{c|}{ aNK +} \\
Untreated & Dinutux & aNK & dinutux \\
\cline { 2 - 4 } Dinutux & 0.003 & $<0.001$ & 0.003 \\
aNK & $\mathrm{x}$ & 0.003 & 0.005 \\
CHLA-255-Fluc & $\mathrm{x}$ & $\mathrm{x}$ & 0.111 \\
Untreated & 0.029 & $<0.001$ & $<0.001$ \\
Dinutux & $\mathrm{x}$ & 0.009 & $<0.001$ \\
aNK & $\mathrm{x}$ & $\mathrm{x}$ & 0.523 \\
\hline
\end{tabular}

Figure 2 (A) Effect of anti-GD2 antibody dinutuximab (dinutux) and human activated natural killer (aNK) cells on human neuroblastoma (NB) cells in vitro. Cytotoxicity assay showing the viability of SMS-KCNR-Fluc and CHLA-255-Fluc human NB cells following 6 -hour treatment with dinutuximab alone $(100 \mathrm{ng} / \mathrm{mL})$, aNK cells alone, or their combination. $1.5 \times 10^{4} \mathrm{NB}$ cells were seeded per well of a 96-well plate along with $1.5 \times 10^{4}$ aNK cells (E:T ratio, 1:1). Each condition contained eight replicates and each experiment was replicated thrice. Comparisons between groups were performed using Student's t-test. (B) Relative GD2 expression on SMS-KCNR-Fluc, CHLA-255-Fluc, and COG-N-415x (PDX) as measured by flow cytometry. Dotted lines, isotype-matched irrelevant antibody control.

The $\mathrm{p}$ values refer to two-sided tests and a $\mathrm{p}$ value $<0.05$ was considered significant.

\section{RESULTS}

Activated natural killer cells in combination with dinutuximab are cytotoxic for human NB cells in vitro

To assess antibody-dependent cell-mediated cytotoxicity (ADCC) of aNK cells in vitro, human NB cells lines SMSKCNR-Fluc and CHLA-255-Fluc were treated with vehicle control, aNK cells alone, dinutuximab alone, or dinutuximab in combination with aNK cells (figure 2A). For both cell lines, treatment with aNK cells at an effector:target (E:T) cell ratio of 1:1 for 6 hours, either in combination with dinutuximab or alone, significantly decreases cell viability compared with no treatment or dinutuximab alone. Increased cytotoxicity of aNK cells combined with dinutuximab compared with aNK cells alone trends toward significance. These findings demonstrate the potent ADCC of aNK cells against NB cells when combined with dinutuximab. GD2 expression was confirmed across the NB cell lines and the patient-derived xenograft by flow cytometry (figure 2B).
Activated natural killer cells plus dinutuximab given before resection of the primary NB tumor decreases tumor burden and prolongs survival

In vivo, we modified our previously described model of metastatic NB in which NB cells (SMS-KCNR-Fluc (MYCN amplified), CHLA-255-Fluc (c-myc overexpressed), or COG-N-415x (MYCN amplified, ALK mutated)) are injected into the left kidney, with the resulting xenograft tumor undergoing surgical resection 19 days later, as opposed to 7 days later. We have observed in previous studies that $100 \%$ of the mice have histopathologic confirmation of metastatic disease in the liver and bone marrow. ${ }^{9}$ To evaluate the effect of the timing of immunotherapy relative to tumor resection, immunotherapy was either initiated 12 days before surgical resection (four treatments before surgery and four treatments after surgery) or delayed until 2 days after resection (eight treatments after surgery). Building on our previous study in which control groups received dinutuximab alone or aNK cells alone to evaluate immunotherapy after surgery, and to determine the effect of surgery, a control group was evaluated that did not undergo resection of the 

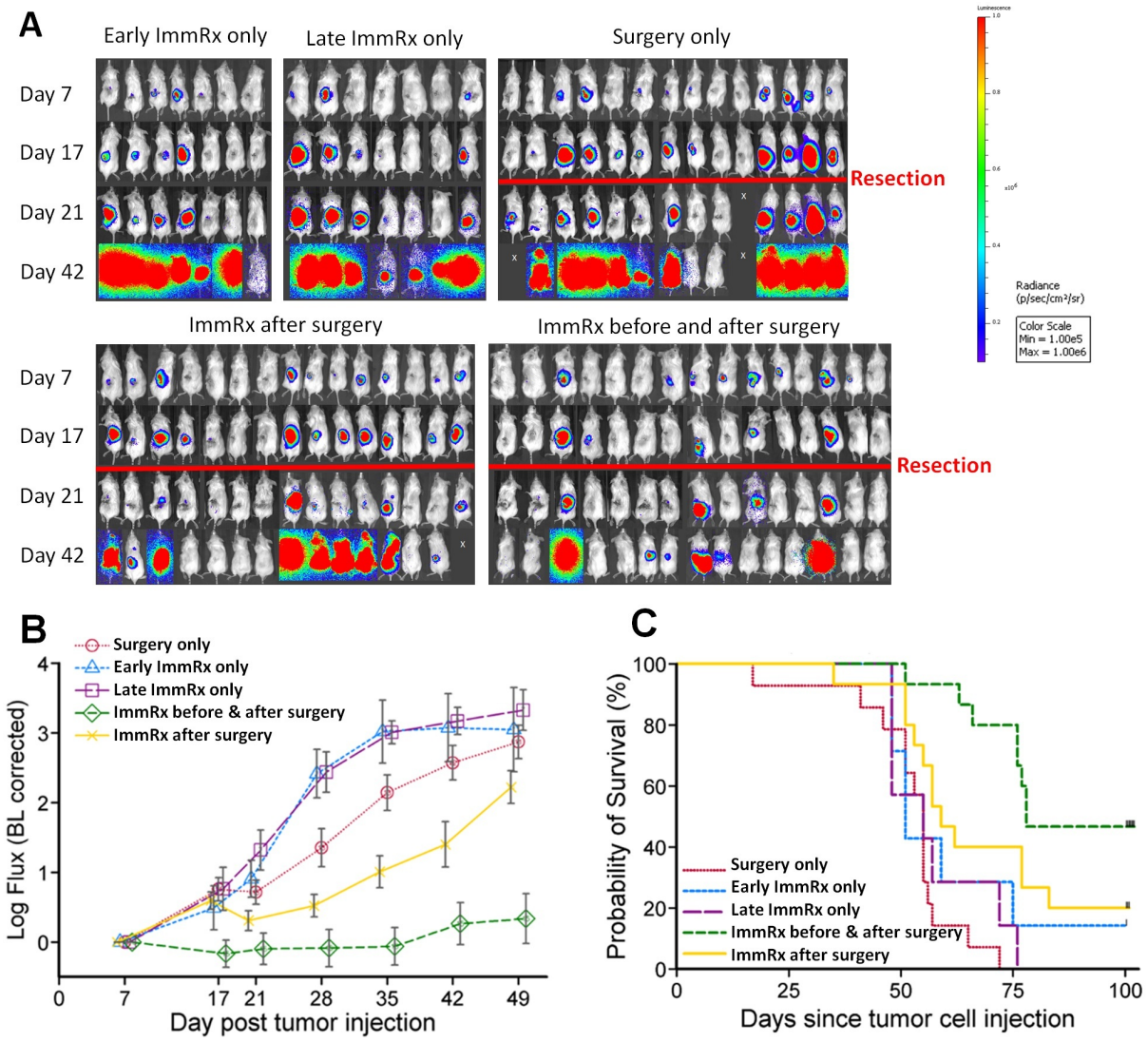

\begin{tabular}{|c|c|c|c|c|}
\multicolumn{5}{|c|}{ Pairwise comparison p-value } \\
\cline { 1 - 1 } Group & Surgery only & $\begin{array}{c}\text { Early ImmRx } \\
\text { only }\end{array}$ & $\begin{array}{c}\text { Late ImmRx } \\
\text { only }\end{array}$ & $\begin{array}{c}\text { ImmRx before } \\
\text { \& after surgery }\end{array}$ \\
\cline { 1 - 4 } $\begin{array}{c}\text { Early ImmRx } \\
\text { only }\end{array}$ & 0.67 & - & - & - \\
\cline { 1 - 1 } $\begin{array}{c}\text { Late ImmRx } \\
\text { only }\end{array}$ & 0.70 & 0.97 & - & - \\
\cline { 1 - 1 } $\begin{array}{c}\text { ImmRx before } \\
\text { \& after surgery }\end{array}$ & $<0.01$ & $<0.01$ & $<0.01$ & - \\
\cline { 1 - 3 } $\begin{array}{c}\text { ImmRx after } \\
\text { surgery }\end{array}$ & $<0.01$ & 0.04 & 0.04 & 0.14 \\
\hline
\end{tabular}

\begin{tabular}{|c|c|c|c|c|}
\hline Group & Surgery only & $\begin{array}{c}\text { Early ImmRx } \\
\text { only }\end{array}$ & $\begin{array}{c}\text { Late ImmRx } \\
\text { only }\end{array}$ & $\begin{array}{c}\text { ImmRx before } \\
\text { \& after surgery }\end{array}$ \\
\cline { 1 - 4 } $\begin{array}{c}\text { Early ImmRx } \\
\text { only }\end{array}$ & 0.62 & -- & -- & - \\
\cline { 1 - 1 } $\begin{array}{c}\text { Late ImmRx } \\
\text { only }\end{array}$ & 0.99 & 0.64 & - & - \\
\cline { 1 - 1 } $\begin{array}{c}\text { ImmRx before } \\
\text { \& after surgery }\end{array}$ & $<0.01$ & $<0.01$ & $<0.01$ & - \\
\cline { 1 - 1 } $\begin{array}{c}\text { ImmRx after } \\
\text { surgery }\end{array}$ & 0.03 & 0.22 & 0.08 & $<0.01$ \\
\hline
\end{tabular}

Figure 3 Effect of activated natural killer (aNK) cells and dinutuximab on disease burden and survival of NOD-scid gamma (NSG) mice implanted with SMS-KCNR-Fluc cells. (A) Bioluminescent images of disease burden (primary tumor and/or disseminated disease) on day $7,17,21$, and 42 after tumor cell implantation for each treatment group. $1 \times 10^{6}$ SMS-KCNR-Fluc cells were injected beneath the capsule of the left kidney of NSG mice. Treatments were given intravenously twice per week for 4 weeks to indicated groups, as per figure 1. Resection, if performed, occurred on day 19. (B) Bioluminescent imaging was performed weekly and tumor flux (photons/s) measurements were used to assess treatment effects. $P$ values for pairwise comparisons of groups. (C) Kaplan-Meier survival plot. P values for pairwise comparisons of groups are shown.

primary tumor but did receive immunotherapy on the same schedule as the experimental groups. ${ }^{4}$ To control for the benefit of immunotherapy, an additional group was included that underwent tumor resection only without immunotherapy.

After intrarenal injection with SMS-KCNR-Fluc cells, tumor growth was monitored by weekly bioluminescent imaging (figure 3A). Mice which received dinutuximab plus aNK cell immunotherapy before surgical resection were observed to have less tumor disease at the time of surgical resection, and by 42 days after surgery, this group had less detectable disease than other treatment groups (figure 3B). Moreover, when mice were followed to day 100, 11 mice were alive without detectable signal, a majority of which received immunotherapy initiated before resection (figure 3C). Mice which received early immunotherapy with surgical resection had significantly increased survival compared with other treatment groups $(\mathrm{p}<0.01)$.

In mice injected with CHLA-255-Fluc cells, bioluminescent imaging suggest that mice treated with dinutuximab and aNK cells initiated prior to surgical resection had smaller primary tumors by the day of surgical resection, day 19 (figure 4A,B). Furthermore, immunotherapy initiated before tumor resection significantly reduced disease burden in mice at later time points compared with the same course of treatment begun after resection $(p=0.02)$ (figure 4B). Survival was significantly increased for mice receiving immunotherapy initiated before surgery compared with those that received immunotherapy only after resection of tumor $(\mathrm{p}=0.04)$ or those that did not receive immunotherapy $(\mathrm{p}<0.01)$ (figure $4 \mathrm{C})$.

In addition, we used a patient-derived xenograft NB tumor model by injection of COG-N-415x PDX cells. The 

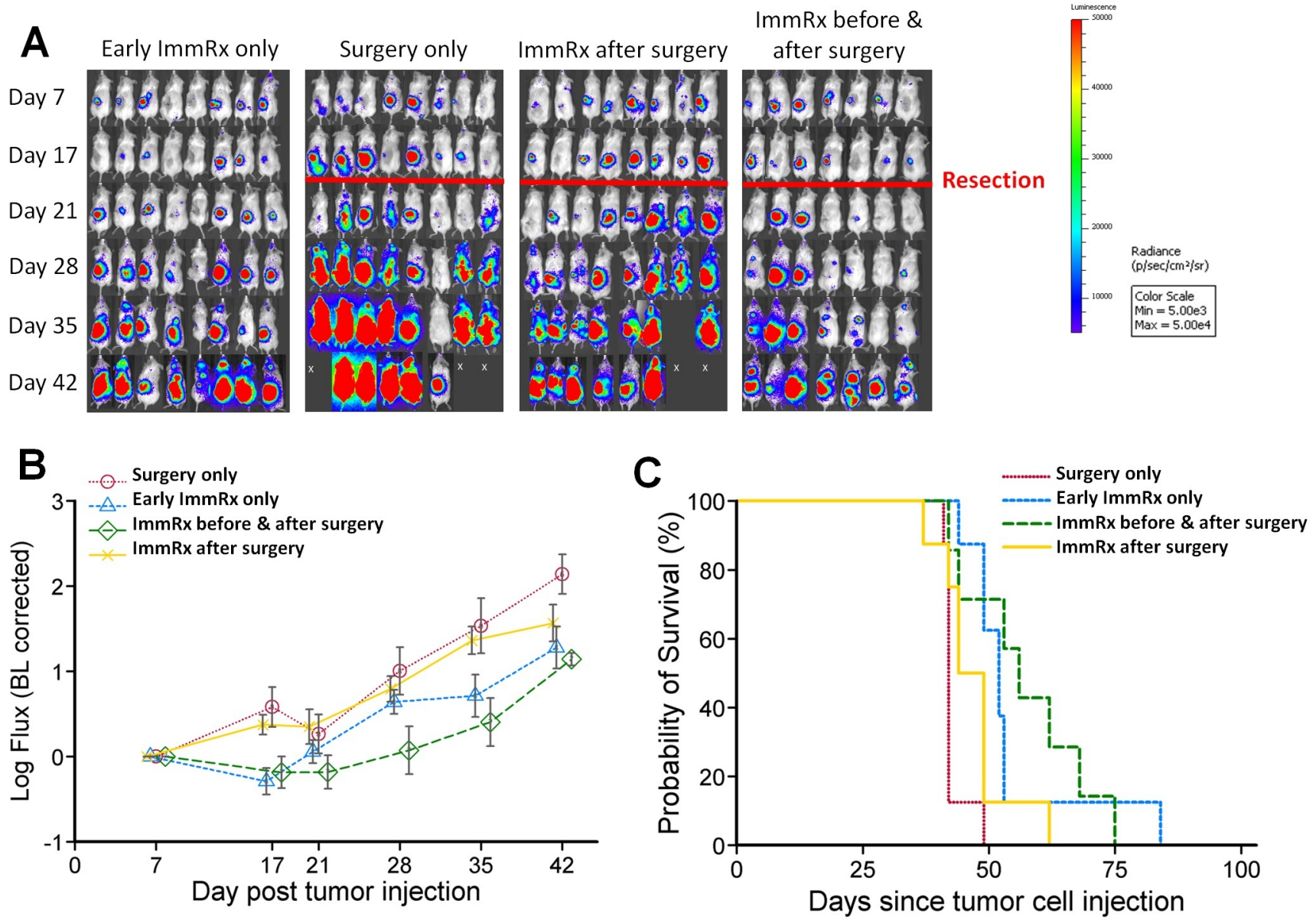

\begin{tabular}{|c|c|c|c|}
\hline \multicolumn{4}{|c|}{ Pairwise comparison p-value } \\
\hline Group & Surgery only & $\begin{array}{l}\text { Early ImmRx } \\
\text { only }\end{array}$ & $\begin{array}{l}\text { ImmRx before } \\
\text { \& after surgery }\end{array}$ \\
\hline $\begin{array}{l}\text { Early ImmRx } \\
\text { only }\end{array}$ & $<0.01$ & -- & -- \\
\hline $\begin{array}{l}\text { ImmRx before } \\
\text { \& after surgery }\end{array}$ & $<0.01$ & 0.34 & -- \\
\hline $\begin{array}{c}\text { ImmRx after } \\
\text { surgery }\end{array}$ & 0.10 & 0.15 & 0.02 \\
\hline
\end{tabular}

\begin{tabular}{|c|c|c|c|}
\hline \multicolumn{4}{|c|}{ Pairwise comparison $\mathrm{p}$-value } \\
\hline Group & Surgery only & $\begin{array}{c}\text { Early ImmRx } \\
\text { only }\end{array}$ & $\begin{array}{l}\text { ImmRx before } \\
\text { \& after surgery }\end{array}$ \\
\hline $\begin{array}{c}\text { Early ImmRx } \\
\text { only }\end{array}$ & 0.01 & -- & -- \\
\hline $\begin{array}{l}\text { ImmRx before } \\
\& \text { after surgery }\end{array}$ & $<0.01$ & 0.59 & -- \\
\hline $\begin{array}{l}\text { ImmRx after } \\
\text { surgery }\end{array}$ & 0.30 & 0.09 & 0.04 \\
\hline
\end{tabular}

Figure 4 In NOD-scid gamma (NSG) mice implanted with CHLA-255-Fluc cells, effect of treatment with activated natural killer (aNK) cells and dinutuximab prior to resection of primary tumor on disease burden and survival. (A) Bioluminescent images of disease burden up to day 42 after neuroblastoma cell implantation. $1 \times 10^{6} \mathrm{CHLA}-255$-Fluc cells were injected as in figure 3 . (B) Bioluminescent imaging was performed weekly and tumor flux (photons/s) measurements were used to assess treatment effects. P values for pairwise comparisons of groups. (C) Kaplan-Meier survival plot. P values for pairwise comparisons of groups are shown. ImmRx, immunotherapy.

PDX cells are not fluorescently labeled, and thus, mice were only monitored for survival. Survival was significantly increased among mice that received dinutuximab and aNK cells prior to resection of the tumor compared with all other groups $(\mathrm{p}<0.01)$ (figure 5).

Together, these in vivo experiments indicate that targeting NB with dinutuximab plus aNK cells prior to surgery leads to smaller tumors at the time of surgical resection, decreased overall disease burden, and increased survival. To evaluate mechanisms affecting the degree of response to therapy, surface immunophenotyping of the NB cells used in this study (CHLA-255-Fluc, SMS-KCNRFluc, and COG-N-415x) was performed, demonstrating a distinct pattern of differences in the level of expression of natural cytotoxicity receptors, immune checkpoint ligands, adenosine pathway surface proteins (CD39, CD73), and major histocompatibility complex (MHC) class I molecules (see online supplemental figure 1).

\section{Activated natural killer cells given with dinutuximab infiltrate primary NB tumors}

To characterize the activity of aNK cells in vivo, we investigated infiltration of aNK cells into established primary tumors after a single intravenous infusion with dinutuximab, which models the current treatment protocol for human patients. Specifically, we measured when aNK cells infiltrated the tumor, when these levels were maximal, and the length of time that aNK cells persisted within the 


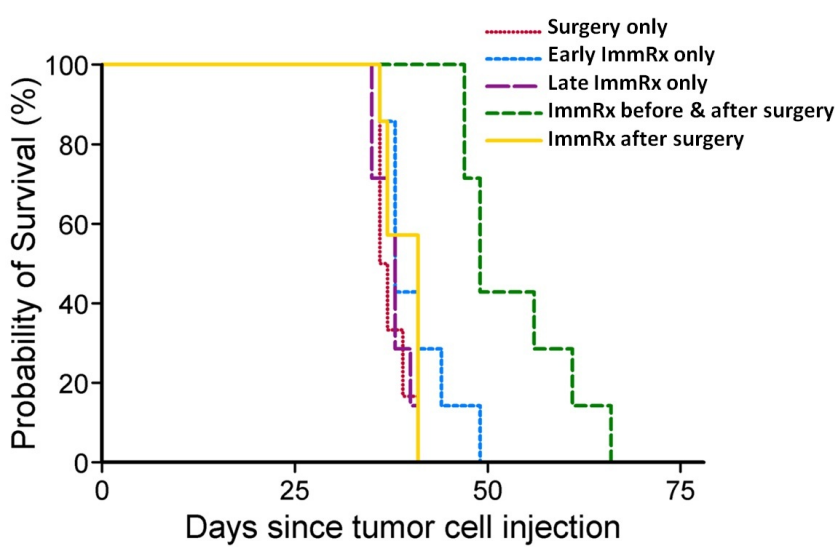

\begin{tabular}{|c|c|c|c|c|}
\hline Group & Surgery only & $\begin{array}{c}\text { Early ImmRx } \\
\text { only }\end{array}$ & $\begin{array}{c}\text { Late ImmRx } \\
\text { only }\end{array}$ & $\begin{array}{c}\text { ImmRx before } \\
\text { \& after surgery }\end{array}$ \\
\cline { 1 - 4 } $\begin{array}{c}\text { Early ImmRx } \\
\text { only }\end{array}$ & 0.22 & -- & -- & - \\
\cline { 1 - 1 } $\begin{array}{c}\text { Late ImmRx } \\
\text { only }\end{array}$ & 0.93 & 0.23 & -- & - \\
\cline { 1 - 1 } $\begin{array}{c}\text { ImmRx before } \\
\text { \& after surgery }\end{array}$ & $<0.01$ & $<0.01$ & $<0.01$ & - \\
\cline { 1 - 2 } $\begin{array}{c}\text { Imm after } \\
\text { surgery }\end{array}$ & 0.50 & 0.55 & 0.55 & $<0.01$ \\
\hline
\end{tabular}

Figure 5 After injection of PDX (COG-N-415x) cells beneath the left renal capsule of NOD-scid gamma (NSG) mice, the survival was monitored following treatment with activated natural killer (aNK) cells and dinutuximab prior to resection of primary tumor. Indicated treatments (dinutuximab, aNK cells, interleukin (IL)-2, IL-15) were given intravenously. KaplanMeier survival plot for mice implanted with PDX xenograft tumors. $\mathrm{P}$ values for pairwise comparisons of groups. ImmRx, immunotherapy.

tumors. At four time points, a single dose of aNK cells was infused since multiple doses may drive ADCC against tumor cells, in turn reducing the number of NB cells and inflating the relative percentage of aNK cells present in the tumor (figure 6A). Flow cytometry of dissociated xenograft tumors demonstrated aNK cell tumor infiltration at 1 day after injection, with the highest concentration observed 5 days after infusion, and aNK cells still present 10 days after injection (figure 6B). Statistical analysis confirmed that the highest percentage of aNK cells was approximately $0.3 \%$ in tumors observed 5 days after injection, suggesting a gradual impact of immunotherapy over time (figure 6C). The presence of NK cells within the xenograft tumor tissue was further confirmed by immunohistochemical staining for the human aNK cell marker CD16 (FcyRIII) (figure 6D). While it is difficult to discern the clinical significance of the population of the infused aNK cells found within the primary tumors of our mouse models, comprising $0.3 \%$ of all cells, it is quite possible that the benefit of aNK cell infusion with anti-GD2 immunotherapy may be due to direct effects on microscopic disseminated tumor cells, rather than on primary tumors.

\section{DISCUSSION}

We hypothesized that immunotherapy with aNK cells plus dinutuximab initiated before resection of primary high-risk NB tumors would reduce disease burden and improve survival. To test this hypothesis, we established intrarenal human NB tumors in immunodeficient NSG mice by injecting SMS-KCNR-Fluc cell lines, CHLA-255Fluc cell lines, or COG-N-415x PDX cells beneath the renal capsule of NSG mice. We showed that infusion of dinutuximab combined with aNK cells, along with IL-2 and IL-15, initiated before primary tumor resection decreases total disease burden and prolongs survival compared with the same treatment initiated after resection. We further demonstrate that aNK cells are present in xenograft tumors 1 day after infusion, maximal at 5 days, and remain present at 10 days.

Our findings highlight the importance of surgical resection of the primary tumor in treating patients with high-risk NB. Mice treated with immunotherapy before and after resection of the primary tumor had greater survival than those receiving the same immunotherapy without surgery. In addition to the benefit of resecting the primary tumor cells, there may be other benefits of surgery including the removal of immunosuppressive tumor-associated macrophages and regulatory $\mathrm{T}$ cells, as well as creation of fewer tumor cell targets for immunotherapy. ${ }^{10}{ }^{11}$ It is well accepted that immunotherapy is most effective against minimal disease, and removal of bulk tumor disease might therefore increase the efficacy of immunotherapy against metastatic disease by multiple mechanisms.

To date, there have been no preclinical studies investigating the efficacy of immunotherapy prior to surgery in pediatric solid tumors. However, preclinical studies of adult cancers have demonstrated potential benefits of preoperative immunotherapy. Using a preclinical mouse model of metastatic breast cancer, Liu et $a l^{12}$ demonstrated improved survival for mice receiving anti-PD-1 and anti-CD25 immunotherapy before resection of the primary tumor as compared with those receiving identical treatments after surgery. In another study using a mouse model of triple negative breast cancer, Brockwell et $a l^{13}$ observed that anti-PD-1 immunotherapy in combination with an interferon inducer administered before primary tumor resection significantly prolonged survival when compared with an identical treatment given only after surgery. Our findings represent the first preclinical data to support immunotherapy prior to surgery for patients with newly diagnosed high-risk NB. The benefit of neoadjuvant immunotherapy may be in targeting microscopic foci of cancer cells prior to resection of the primary tumor. Furthermore, enhancement of antitumor immune responses, such as antitumor NK cell activity, has the potential to reduce metastatic disease responsible for postsurgical recurrence.

Initiation of immunotherapy before surgery, also referred to as 'neoadjuvant immunotherapy', has been investigated in clinical trials, many of which focus on immune checkpoint inhibitors. ${ }^{14}$ In a clinical trial for non-small-cell lung cancer, two preoperative doses of PD-1 blockade administered over 4 weeks prior to 


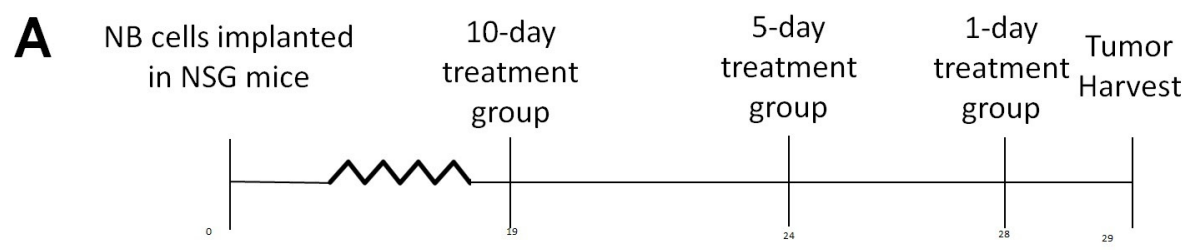

Days after Tumor Cell Implantation

B
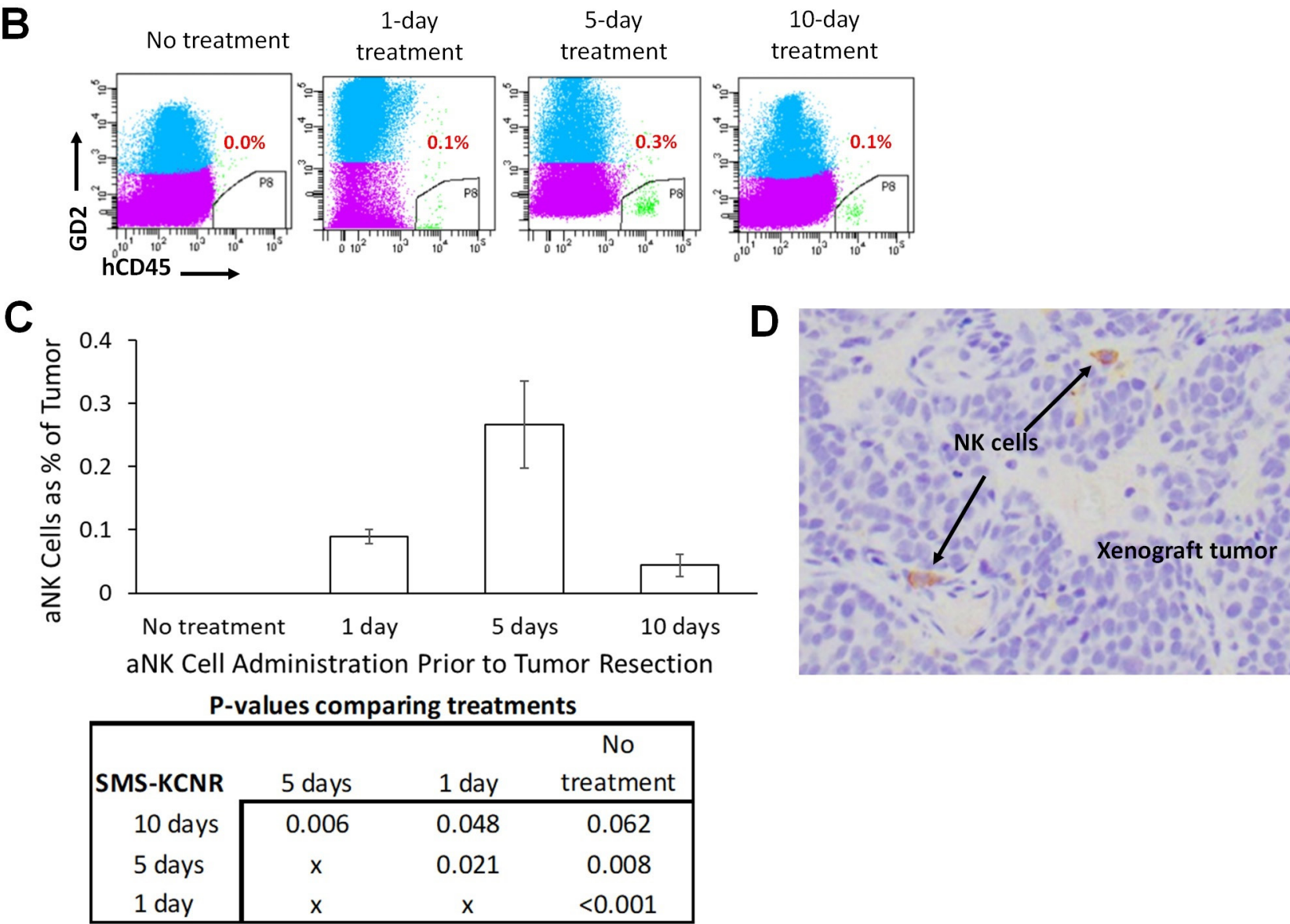

\begin{tabular}{|cccc|}
\multicolumn{4}{c}{ P-values comparing treatments } \\
\hline & & & No \\
SMS-KCNR & 5 days & 1 day & treatment \\
\cline { 2 - 4 } 10 days & 0.006 & 0.048 & 0.062 \\
5 days & $\mathrm{x}$ & 0.021 & 0.008 \\
1 day & $\mathrm{x}$ & $\mathrm{x}$ & $<0.001$ \\
\hline
\end{tabular}

Figure 6 Activated natural killer (aNK) cell infiltration into xenograft neuroblastoma tumors in NOD-scid gamma (NSG) mice. (A) Schematic diagram demonstrating the in vivo experimental model. SMS-KCNR-Fluc neuroblastoma cells were implanted beneath the left renal capsule in NSG mice on day 0 . Mice were treated by intravenous infusion with one dose of dinutuximab $(15 \mu \mathrm{g})$ and $1 \times 10^{7}$ aNK cells plus interleukin (IL)-2 $(2 \mu \mathrm{g})$ and IL-15 $(5 \mu \mathrm{g}) 10$ days, 5 days, 2 days, or 1 day prior to harvest of the xenograft tumor. Control mice did not receive aNK cells prior to tumor harvest. (B) Representative flow cytometry plots demonstrating human NK cells (CD45+) within the xenografts. Harvested tumors were dissociated into single-cell suspensions and analyzed by flow cytometry for expression of GD2 (conjugated to APC) and human CD45 (Alexa Fluor 488). Percentages indicate the frequency of NK cells relative to total cells. (C) Statistical analysis (Student's t-test) of frequencies of the human NK cells in the xenograft tumors shown in (B). 'No treatment group indicates the untreated control group. (D) Immunohistochemistry staining of NK cells within a representative xenograft tumor. NK cells are stained (brown) by anti-human CD16.

surgical resection induced a major pathologic response in $45 \%$ of primary tumors. ${ }^{15}$ In patients with a pathologic response, defined as a tumor with $\leq 10 \%$ of residual viable tumor cells identified by routine H\&E staining, T-cell clones identified by T-cell receptor DNA sequencing were found in the primary tumor and the same clones were found in peripheral blood, suggesting that this immunotherapy might enhance systemic antitumor $\mathrm{T}$ cell activity that could potentially reduce metastatic disease which is primarily responsible for postsurgical recurrence. While no preclinical studies of neoadjuvant immunotherapy have been completed for pediatric solid tumors, a clinical study by Furman $e t a l^{16}$ evaluated administration of humanized anti-GD2 immunotherapy with induction chemotherapy. ${ }^{13}$ The authors report that at least a partial response or better was noted in $76 \%$ of patients, and that the treatment resulted in reduction in both tumor volume and semiquantitative 123I-metaiodobenzylguanidine scoring, thus supporting the future exploration of immunotherapy prior to surgical resection. No data were presented regarding whether patients progressed to resection and outcomes thereafter. An ongoing pilot clinical 
study by the Children's Oncology Group (NCT03786783) is investigating the benefit of adding dinutuximab and GM-CSF to standard induction chemotherapy for patients with newly diagnosed NB. While this clinical study is not investigating the benefit of aNK cell infusion, nor is it specifically examining the role of surgery, the addition of dinutuximab immunotherapy during induction and prior to surgical resection will test whether the earlier administration of immunotherapy will improve outcomes. ${ }^{17}$

One potential concern with administering immunotherapy before resection would be a probable delay in surgery. In the current clinical trial NANT 2013-01, a single dose of aNK cells is infused intravenously after 4 days of continuous dinutuximab infusion, followed by 23 days of rest before beginning each cycle. The 28-day cycles were designed to limit antibody-related toxicity, such as myelosuppression and neuropathy. This parallels the current standard dosing regimen of dinutuximab alone, which also occurs over 4 days in 28-day cycles. If only one aNK treatment is planned preoperatively, our data suggest that aNK cell concentration peaks before 10 days after infusion, and thus a delay in resection to allow for maximal effect may not be lengthy. If multiple preoperative aNK treatments were administered, a lengthier delay of surgery may be required; however, the benefit of preoperative immunotherapy may outweigh this delay, as suggested by our current and previous study. ${ }^{4}$

While our study supports the initiation of immunotherapy prior to surgical removal of the primary tumor, our experimental model has limitations. First, NSG mice lack their own NK, B, and T cells, and have defective dendritic cells and macrophages because of alleles in the NOD/ShiLt genetic background. Together, these defects preclude effective innate and adaptive murine immune responses against the injected human tumor cells, even in the presence of dinutuximab. The lack of a reproducible effect of the combination of dinutuximab plus adoptive aNK cell therapy in the absence of additional therapy (such as surgery or other modalities) in our in vivo models may be due to these defects and is consistent with our previous reports. ${ }^{46}$ Importantly, this limitation in our experimental model is in contrast with the efficacy of repeated courses of dinutuximab in the clinic. ${ }^{1}$ Despite the above-mentioned defects in the NSG model, our findings suggest that in immune-competent human patients, stimulation of NK cells with dinutuximab and/or infusion of autologous ex vivo-expanded aNK cells before surgery could enhance patients' innate and adaptive immune responses via increased release of proinflammatory cytokines such as interferon-y from the activated NK cells.

Another limitation of our model involves the potential for suppression of aNK cytotoxic function by interactions between killer cell immunoglobin-like receptors (KIRs) and HLA class I molecules. Expression of KIRs on NK cells differs between human subjects, and thus the clinical response to immunotherapy with aNK cells and dinutuximab may vary. Moreover, expression of HLA molecules differs between tumors, reflecting the heterogeneity of NB disease and potentially leading to variability in response to immunotherapy. These physiologically relevant interactions cannot be accurately replicated in our model given that the aNK cells and NB cells used were obtained from different human donors. However, these factors may contribute to the differences observed between the various cell lines from our in vivo studies. Other contributors to the varying response to immunotherapy from our animal studies may include differential expression of natural cytotoxicity receptors, immune checkpoint ligands, and HLA-E on NB cells (see online supplemental figure 1) and possibly other pathways not examined in this study. However, previous in vitro work has demonstrated that such activating signals for aNK cells can at least partially overcome inhibitory signals. ${ }^{18}$ Furthermore, human NB cells rarely express classical HLA class I molecules on their surface suggesting that the kinetics of KIR:MHC interactions may be dynamic, following a single monthly dose of dinutuximab (whether with or without aNK cells) and may depend on induction of HLA expression by infiltrating immune cells producing IFN- $\gamma$ (see online supplemental figure 1). ${ }^{19}{ }^{20}$ Another described mechanism of cellular cytotoxicity of NK cells is through the activation of death receptor-mediated apoptosis. However, in our study, we did not investigate the role of death receptors, as we have previously demonstrated that nearly all of the cytotoxicity of activated NK cells against NB cells can be abrogated by concanamycin A. This finding suggests that the mechanism of cytotoxicity is primarily due to the release of perforin and granzymes, while death receptor-mediated cytotoxicity contributes a minor role to cytotoxicity of aNK cells against NB cells. ${ }^{8}$

In a previous study, we reported that adoptive transfer of aNK cells plus dinutuximab increases survival of NOD/ SCID mice without additional therapy. ${ }^{3}$ However, the NOD/SCID mice used in that study are less immunosuppressed than NSG mice and retain some macrophage function, which may help mediate and augment the effects of dinutuximab. Therefore, comparison of results between the two studies and between NSG versus NOD/ SCID mice is difficult. In a subsequent report, we showed small but detectable increases in survival of NSG mice injected with CHLA-255-Fluc and COG-N-415x cells (and a larger increase in survival of mice injected with CHLA136-Fluc cells which are not in the present manuscript) when administration of aNK cells and dinutuximab followed surgery on day 7 after tumor cell injection. ${ }^{4}$ In contrast, in the current study, a significant increase in survival when immunotherapy followed surgery on day 19 after tumor cell injection was observed only in mice injected with SMS-KCNR-Fluc cells but not with CHLA255-Fluc or COG-N-415x cells. The differences in survival of mice between the findings of Barry $e t a l^{4}$ versus our current findings may be attributed to the fact that the present study administered immunotherapy to mice with larger and more established tumors following resection on day 19, whereas the prior work administered immunotherapy to smaller, less well-established tumors following 
resection on day 7 . The differences between survival of mice injected with CHLA-255-Fluc and COG-N-415x cells in Barry $e t a l^{4}$ versus the current study may also be attributed, at least in part, to differences in expression of natural cytotoxicity receptors/ligands, immune checkpoint receptors/ligands, and KIR and HLA alleles in the disparate donors of NK cells and tumors. Despite differences in timing of tumor resection and immunotherapy, and in human aNK cell donors, our current manuscript provides strong evidence that administration of dinutuximab plus aNK cells is superior when it precedes rather than follows surgery.

In summary, we demonstrate with an immunodeficient mouse model of human NB that initiation of immunotherapy with dinutuximab plus activated NK cells prior to tumor resection enhances control of disease and prolongs survival as compared with identical therapy initiated after surgery. We show for the first time that adoptively transferred human activated NK cells can infiltrate the primary tumor within 1 day after infusion and can be present in greatest numbers after 5 days. These data provide support for clinical testing of activated NK cells in combination with dinutuximab as part of induction therapy, prior to resection of primary tumor, in children with high-risk NB.

\section{Author affiliations}

${ }^{1}$ Division of Pediatric Surgery, Department of Surgery, Children's Hospital Los Angeles, Los Angeles, California, USA

${ }^{2}$ Division of Hematology, Oncology, Blood and Marrow Transplantation, Department of Pediatrics, Children's Hospital Los Angeles, Los Angeles, California, USA ${ }^{3}$ Department of Pathology and Laboratory Medicine, Children's Hospital Los Angeles, Los Angeles, California, USA

${ }^{4}$ Department of Surgery, Keck School of Medicine, University of Southern California, Los Angeles, California, USA

Twitter Michael John Zobel @Michael_Zobel, Abigail K Zamora @akzamora7, Danny Lascano @drdlascano and Eugene S Kim @dreskim

Funding The authors have not declared a specific grant for this research from any funding agency in the public, commercial or not-for-profit sectors.

\section{Competing interests None declared.}

Patient consent for publication Not required.

Ethics approval All animal experiments were performed in accordance with a protocol approved by the Institutional Animal Care and Use Committee of Children's Hospital Los Angeles (IACUC \#363-17).

\section{Data availability statement Data are available upon reasonable request.}

Supplemental material This content has been supplied by the author(s). It has not been vetted by BMJ Publishing Group Limited (BMJ) and may not have been peer-reviewed. Any opinions or recommendations discussed are solely those of the author(s) and are not endorsed by BMJ. BMJ disclaims all liability and responsibility arising from any reliance placed on the content. Where the content includes any translated material, BMJ does not warrant the accuracy and reliability of the translations (including but not limited to local regulations, clinical guidelines, terminology, drug names and drug dosages), and is not responsible for any error and/or omissions arising from translation and adaptation or otherwise.

Open access This is an open access article distributed in accordance with the Creative Commons Attribution Non Commercial (CC BY-NC 4.0) license, which permits others to distribute, remix, adapt, build upon this work non-commercially, and license their derivative works on different terms, provided the original work is properly cited, appropriate credit is given, any changes made indicated, and the use is non-commercial. See http://creativecommons.org/licenses/by-nc/4.0/.

\section{ORCID iDs}

Michael John Zobel http://orcid.org/0000-0002-8082-512X

Michael A Sheard http://orcid.org/0000-0002-4960-7650

Eugene S Kim http://orcid.org/0000-0001-7959-5614

\section{REFERENCES}

1 Yu AL, Gilman AL, Ozkaynak MF, et al. Anti-Gd2 antibody with GMCSF, interleukin-2, and isotretinoin for neuroblastoma. N Engl J Med 2010;363:1324-34.

2 Armideo E, Callahan C, Madonia L. Immunotherapy for high-risk neuroblastoma: management of side effects and complications. $J$ Adv Pract Oncol 2017;8:44-55.

3 Liu Y, Wu H-W, Sheard MA, et al. Growth and activation of natural killer cells ex vivo from children with neuroblastoma for adoptive cell therapy. Clin Cancer Res 2013;19:2132-43.

4 Barry WE, Jackson JR, Asuelime GE, et al. Activated natural killer cells in combination with anti-GD2 antibody Dinutuximab improve survival of mice after surgical resection of primary neuroblastoma. Clin Cancer Res 2019;25:325-33.

5 Tran HC, Wan Z, Sheard MA, et al. Tgf $\beta r 1$ blockade with galunisertib (LY2157299) enhances anti-neuroblastoma activity of the anti-GD2 antibody dinutuximab (ch14.18) with natural killer cells. Clin Cancer Res 2017;23:804-13.

$6 \mathrm{Wu} \mathrm{H}-\mathrm{W}$, Sheard MA, Malvar J, et al. Anti-CD105 antibody eliminates tumor microenvironment cells and enhances anti-GD2 antibody immunotherapy of neuroblastoma with activated natural killer cells. Clin Cancer Res 2019;25:4761-74.

7 Denman CJ, Senyukov VV, Somanchi SS, et al. Membrane-bound IL-21 promotes sustained ex vivo proliferation of human natural killer cells. PLoS One 2012; 7:e30264.

8 Sheard MA, Asgharzadeh S, Liu Y, et al. Membrane-bound TRAIL supplements natural killer cell cytotoxicity against neuroblastoma cells. J Immunother 2013;36:319-29.

9 Jackson JR, Kim Y, Seeger RC, et al. A novel minimal residual disease model of neuroblastoma in mice. J Pediatr Surg 2016;51:991-4.

10 Guisier $\mathrm{F}$, Cousse S, Jeanvoine $\mathrm{M}$, et al. A rationale for surgical debulking to improve anti-PD1 therapy outcome in non small cell lung cancer. Sci Rep 2019;9:16902.

11 Asgharzadeh S, Salo JA, Ji L, et al. Clinical significance of tumorassociated inflammatory cells in metastatic neuroblastoma. $J$ Clin Oncol 2012;30:3525-32.

12 Liu J, Blake SJ, Yong MCR, et al. Improved efficacy of neoadjuvant compared to adjuvant immunotherapy to eradicate metastatic disease. Cancer Discov 2016;6:1382-99.

13 Brockwell NK, Owen KL, Zanker D, et al. Neoadjuvant interferons: critical for effective PD-1-Based immunotherapy in TNBC. Cancer Immunol Res 2017;5:871-84.

14 Topalian SL, Taube JM, Pardoll DM. Neoadjuvant checkpoint blockade for cancer immunotherapy. Science 2020;367. doi:10.1126/ science.aax0182. [Epub ahead of print: 3101 2020].

15 Forde PM, Chaft JE, Smith KN, et al. Neoadjuvant PD-1 blockade in resectable lung cancer. N Engl J Med 2018;378:1976-86.

16 Furman WL, Federico SM, McCarville MB, et al. A phase II trial of Hu14.18K322A in combination with induction chemotherapy in children with newly diagnosed high-risk neuroblastoma. Clin Cancer Res 2019;25:6320-8.

17 Neuroblastoma Treatment $(\mathrm{PDQ}(\mathrm{R}))$. Health profession version. $P D Q$ cancerInformation summaries. Bethesda, MD: National Cancer Institute (US), 2020.

18 Zhang H, Cui Y, Voong N, et al. Activating signals dominate inhibitory signals in CD137L/IL-15 activated natural killer cells. J Immunother 2011;34:187-95.

19 Lampson LA, Fisher CA, Whelan JP. Striking paucity of HLA-A, B, $\mathrm{C}$ and beta 2-microglobulin on human neuroblastoma cell lines. $J$ Immunol 1983;130:2471-8.

20 Favrot MC, Combaret V, Goillot E, et al. Expression of leucocyte adhesion molecules on 66 clinical neuroblastoma specimens. Int $J$ Cancer 1991;48:502-10. 